\title{
Optimising the Collinear Resonance Ionisation Spectroscopy (CRIS) experiment at CERN-ISOLDE
}

\author{
A.R. Vernon ${ }^{\mathrm{a}, *}$, R.P. de Groote ${ }^{\mathrm{f}, \mathrm{d}}$, J. Billowes ${ }^{\mathrm{a}}$, C.L. Binnersley ${ }^{\mathrm{a}}$, T.E. Cocolios ${ }^{\mathrm{d}}$, G. J. Farooq-Smith ${ }^{\mathrm{d}}$, K.T. Flanagan ${ }^{\mathrm{a}, \mathrm{c}}$, \\ R.F. Garcia Ruiz ${ }^{\mathrm{a}, \mathrm{b}}$, W. Gins ${ }^{\mathrm{d}}$, Á. Koszorús ${ }^{\mathrm{d}}$, G. Neyens ${ }^{\mathrm{d}}$, C. M. Ricketts ${ }^{\mathrm{a}}$, A.J. Smith ${ }^{\mathrm{a}}$, S.G. Wilkins ${ }^{\mathrm{a}, 1}$, X.F. Yang ${ }^{\mathrm{f}, \mathrm{e}}$ \\ ${ }^{a}$ School of Physics and Astronomy, The University of Manchester, Manchester, M13 9PL, United Kingdom \\ ${ }^{b}$ ISOLDE, EP Department, CERN, CH-1211 Geneva-23, Switzerland \\ ${ }^{c}$ Photon Science Institute Alan Turing Building, University of Manchester, Manchester M13 9PY \\ ${ }^{d}$ KU Leuven, Instituut voor Kern- en Stralingsfysica, B-3001 Leuven, Belgium \\ ${ }^{e}$ School of Physics and State Key Laboratory of Nuclear Physics and Technology, Peking University, Beijing 100871, China \\ ${ }^{f}$ Department of Physics, University of Jyväskylä, Survontie 9, Jyväskylä, FI-40014, Finland
}

\begin{abstract}
The CRIS experiment at CERN-ISOLDE is a dedicated laser spectroscopy setup for high-resolution hyperfine structure measurements of nuclear observables of exotic isotopes. Between 2015 and 2018 developments have been made to improve the background suppression, laser-atom overlap and automation of the beamline. Furthermore, a new ion source setup has been developed for offline studies. Here we present the latest technical developments and future perspectives for the experiment.
\end{abstract}

Keywords: Collinear resonance ionization spectroscopy; CRIS; CERN-ISOLDE; laser spectroscopy; high-resolution; hyperfine structure.

\section{Introduction}

The Collinear Resonance Ionisation Spectroscopy (CRIS) ${ }_{30}$ technique $[1,2]$ combines the high-resolution of collinear fast beam laser spectroscopy $[3,4]$, with the high selectivity and 5 detection efficiency of multi-step ionization, needed to study isotopes far from stability. These measurements allow nuclear model independent studies of the electromagnetic properties of exotic isotopes, such as the nuclear spin, nuclear magnetic dipole and electric quadrupole moments as well as changes in 10 mean squared charge radii [5]. States with half-lives as short as $\mathrm{t}_{1 / 2}=5 \mathrm{~ms}\left({ }^{214} \mathrm{Fr}\right)$ have been measured using this technique [6].

Since 2015 there have been 8 successful experimental campaigns at CRIS measuring exotic isotopes across the nuclear chart. In the heavy mass region this includes measurements 15 of the nuclear electromagnetic and decay properties of ${ }^{203} \mathrm{Fr}$ [7], ${ }^{206} \mathrm{Fr}[8]$ and ${ }^{231} \mathrm{Ra},{ }^{233} \mathrm{Ra}$ [9]. Where ${ }^{203} \mathrm{Fr}$ was measured with atomic transition linewidth of $25 \mathrm{MHz}$ [7]. While in the medium-mass region, measurements of ${ }^{65-82} \mathrm{Ga}[10]$ and ${ }^{73-78} \mathrm{Cu}[11]$ were made, with demonstrated sensitivities below $2020 \mathrm{ion} / \mathrm{s}$ for ${ }^{78} \mathrm{Cu}$. The most recent campaigns in the $Z=50$ region have resulted in measurements of ${ }^{101-131} \mathrm{In}$ and ${ }^{104-120} \mathrm{Sn}$, and of the lightest radioactive isotope measurements at CRIS to date, in the ${ }^{38-52} \mathrm{~K}$ region.

Optimal extraction of nuclear observables from these atomic

25 systems has required the development of multi-step laser ionization schemes, which has been made possible by an expanding pool of lasers. See the contribution by Á. Koszorús in these

*adam.vernon@cern.ch; adam.vernon-2@postgrad.manchester.ac.uk same proceedings [12], which presents the systematics of resonant ionisation. The present contribution describes the developments of the CRIS setup and its ion source arrangement, which have underpin these recent measurements. This includes improvements to the background suppression, laser-atom overlap and automation of the beamline. A significant extension to the range of elements available from the CRIS ion source for highresolution measurements is presented. These changes have improved the sensitivity, efficiency, function and versatility of the CRIS setup.

\section{The CRIS beamline}

A schematic of the CRIS beamline is shown in Figure 1. A $33^{\circ}$ deflector is used to overlap radioactive ion beams, delivered from ISOLDE following mass separation [13] and bunching in the gas-filled linear Paul trap ISCOOL [14], with resonant and non-resonant laser beams. To allow laser spectroscopy on the atom, the ion beam is neutralised by an alkali metal vapour contained in a charge-exchange cell (CEC) [15]. The non-neutral fraction is then deflected, and after three differential pumping apertures the lasers are overlapped collinearly with the atom bunch in a region of UHV (referred to as the interaction region).

The frequency of the resonant laser step is scanned, allowing an electronic transition from a lower to an upper state. Scanning the laser frequency rather than Doppler shifting the resonance frequency by sweeping the beam energy avoids the need to compensate for steering of the ion beam with the latter approach [16]. In order to achieve sub-GHz linewidths, light from 55 a pulsed Ti:Sapphire injection-seeded laser (20 MHz linewidth) [17] or alternatively chopped light from a continuous-wave laser 


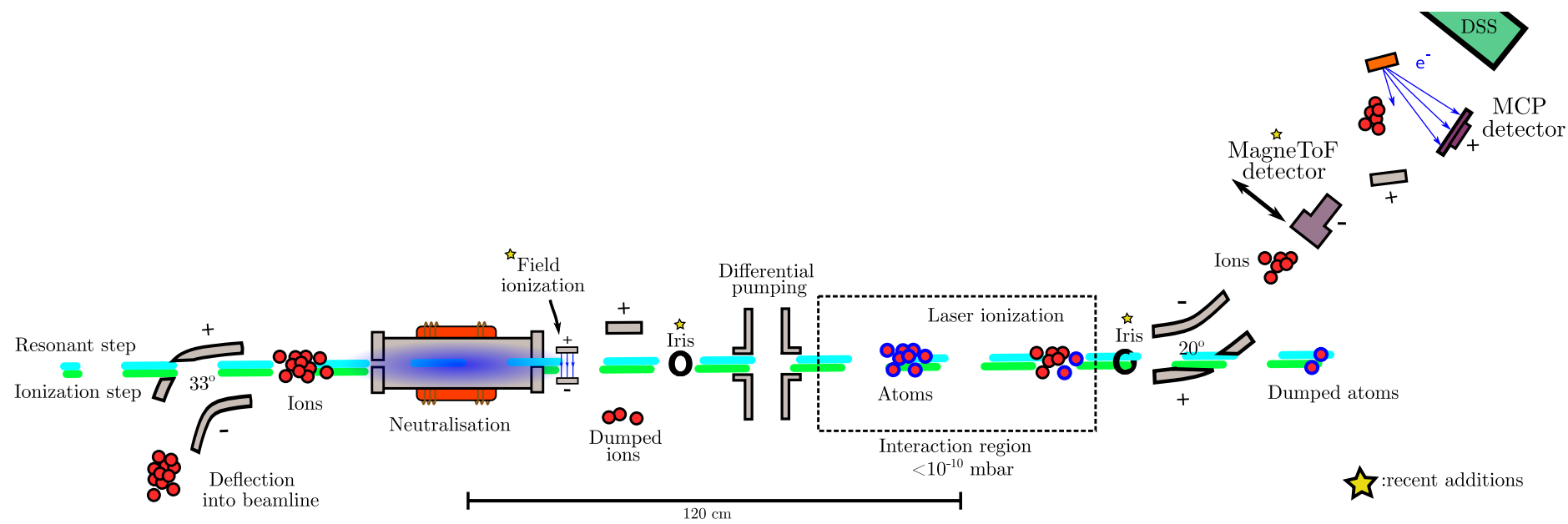

Figure 1: Schematic of the present collinear resonance ionisation spectroscopy set-up at CERN-ISOLDE. Asterisks highlight new additions to the beamline.

light [18] is used. Linewidths down to $25 \mathrm{MHz}$ have been obtained with chopped continuous-wave laser light [7]. Light from a Nd:YAG laser, at a harmonic which exceeds the ionization potential of the atom of interest, is then used as a nonresonant step to ionize the resonantly-excited atoms.

Since 2018, ions are subsequently detected using a ETP DM291 MagneTOF ${ }^{\mathrm{TM}}$ detector. Due to its construction and variable gain, it allows direct ion beam detection with a large 65 dynamic range from single ions to $\mu \mathrm{A}$ beam currents. The ability to tune directly onto the detector has considerably improved the performance of the CRIS setup. Previously a micro-channel plate (MCP) detector was used in its place $[1,10,11]$. However the direct implantation of intense radioactive ion beams resulted 70 in a shorter useful lifetime of the detector and increased background rate, compared to the MagneToF detector. The vertical detector position is now also adjustable in-situ. The installation of irises before and after the interaction region has improved the collinear alignment of the atom and laser beams. The irises also 75 act as apertures for differential pumping, improving the vacuum in the interaction region, with an aperture diameters reducible down to the laser and atom beam widths. A rendering of the iris and actuation system is shown in Figure 4. Both of these 95 additions have improved the ease and reproducibility of setting 80 up experiments.

A second MCP is also used with a conversion dynode for indirect detection of ions by secondary electrons, the ions can be directed onto the conversion dynode en route to the CRIS de-100 cay spectroscopy station (DSS). The CRIS DSS [19], is located 85 at the end of the CRIS beamline and allows for laser-assisted nuclear decay spectroscopy measurements of the highly-pure re-ionized beam [20]. The DSS has been improved with tape station and $\beta$ detection setups, which have extended the decay modes available for tagging, allowing decay-assisted laser spectroscopy. This technique allows for the large background associated with non-resonant ionisation from stable isobaric contaminants to be removed. The $\beta$ detection setup was used for the first time to measure ${ }^{52} \mathrm{~K}[12,21]$.

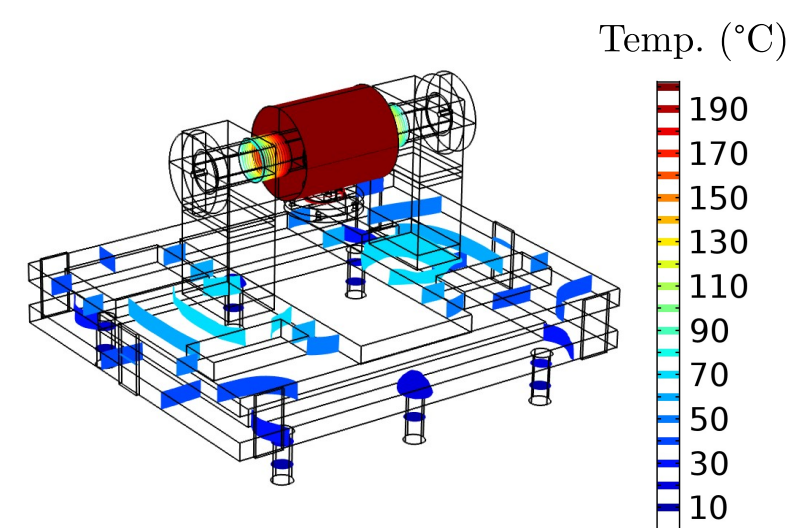

Figure 2: A rendering of the charge-exchange cell and mounting system. Isothermal contours are superimposed for a central heating temperature of 200 ${ }^{\circ} \mathrm{C}$. The upper plate is adjustable by 3 actuators. The vacuum chamber has vertical and horizontal degrees of freedom. The thermal simulation was performed with COMSOL Multiphysics ${ }^{\circledR}$.

The previous CEC was replaced in 2017 with a new UHV compatible chamber and cell. See Figure 2 for a schematic rendering of the design. It has an operation temperature of up to $450^{\circ} \mathrm{C}$ and uses a 5-axis adjustable mounting method, allowing in-situ alignment with smaller transmission apertures. When loaded with $\mathrm{K}$ and the ends heated to below $150{ }^{\circ} \mathrm{C}$, by cartridge heaters at its centre, the cell can be passively cooled and has demonstrated stability for $>200$ hours. Active cooling of the stands of the cell with air has also been incorporated. 


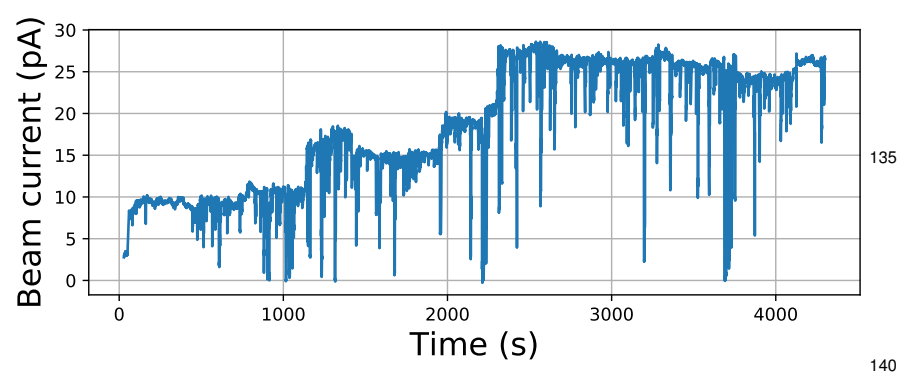

Figure 3: Optimization of transmission through CRIS by Faraday cup current, using the robust conjugate direction search algorithm [22].

Improvements have also been made to increase automation of the experiment. Pneumatic solenoid valves allow the actua-145 tion of Faraday cups and gate valves under the control of a Numato lab's URMC16 16-channel relay controller via USB. A 16-channel Keithley 7001 allows the Faraday cup signals to be switched and measured by a Keithley 6400 picoammeter. Automatic beam transport optimization is performed using the con-150 jugate direction search algorithm developed at SLAC for online optimization [22], implemented in Python. The algorithm is gradient-free and has a high robustness to noise on the signal to be optimized. It can be performed with the signal from Faraday cups or the MagneTOF ${ }^{\mathrm{TM}}$ detector (when used in low gain ${ }^{155}$ mode with the picoammeter), saving crucial person-hours. Potentials are applied to the beamline ion optics using $\pm 6 \mathrm{kV}$ and $\pm 10 \mathrm{kV}$ HTP-EHS HV power supplies from ET-System electronic $\mathrm{GmbH}$. Figure 3 shows an example optimization loop on a Faraday cup, located at the end of the interaction region.

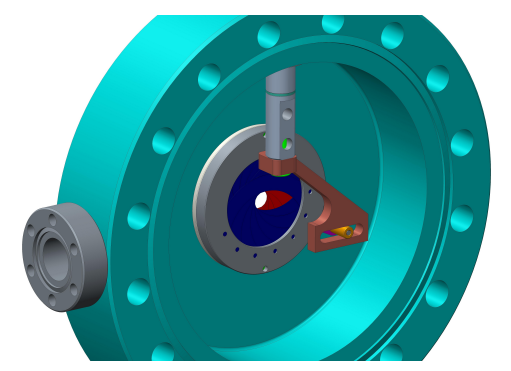

Figure 4: A rendering of the iris and feed-through actuator setup. An additional feedthrough shown on the left side allows measurement of ion beam current onto the iris.

\section{Control software and data acquisition systems}

As the CRIS beamline is continually modified and upgraded, a robust, user-friendly and modular control software is required. As new hardware is introduced into the laboratory, the functionality of this software is expanded. Currently, the CRIS Tuning, Acquisition and Logging (CRISTAL) program controls and monitors i) several different lasers, ii) optical photodiodes, iii) a Fabry-Pérot interferometer, iv) two wave-meters, v) beamline power supplies, vi) ion detectors, vii) temperature readouts, and other devices. The control of these devices, and the data stream they generate, all accessible from anywhere in the laboratory via a student-built network interface protocol.
Figure 5 shows a diagram of the software architecture. Computers that are connected to different equipment run simple scripts that interface with the hardware. These programs can be given instructions and can be polled for data through a message-based network protocol. For this purpose, an intermediate server layer consisting of two server programs were developed. One server focuses entirely on polling all the hardware for which it has the network address, while the other server can send and receive simple instructions. The third layer is the user-interface layer, which consists of graphical user interfaces or web programmes that exchange information with the two server programs. This architecture thus guarantees that all processes and data streams stay organized and synchronized, and furthermore exposes key controls or data streams to the users. All source code is written in Python and is available upon request ${ }^{1}$.

A recent addition to the acquisition hardware of the CRIS experiment is a TimeTagger4-2G, a low cost, mid resolution time-to-digital converter. This 4-channel TDC is PCIe-based and has a precision of $500 \mathrm{ps}$. With this card, the time-of-flight of ions detected by up to four particle detectors can now be recorded precisely, eliminating the need for discrimination and coincidence modules. Thus, precise time-of-flight gates can be made online, during experiments, as well as during offline analysis, and systematic effects related to e.g. detector saturation can be investigated in detail.

Furthermore, the energy spread of the ion beam can be monitored and even Doppler-corrected by correlating the time-offlight information to the laser wavelength [23]. This is in particular important when using ion sources with large energy spread, such as the ablation source presented below.

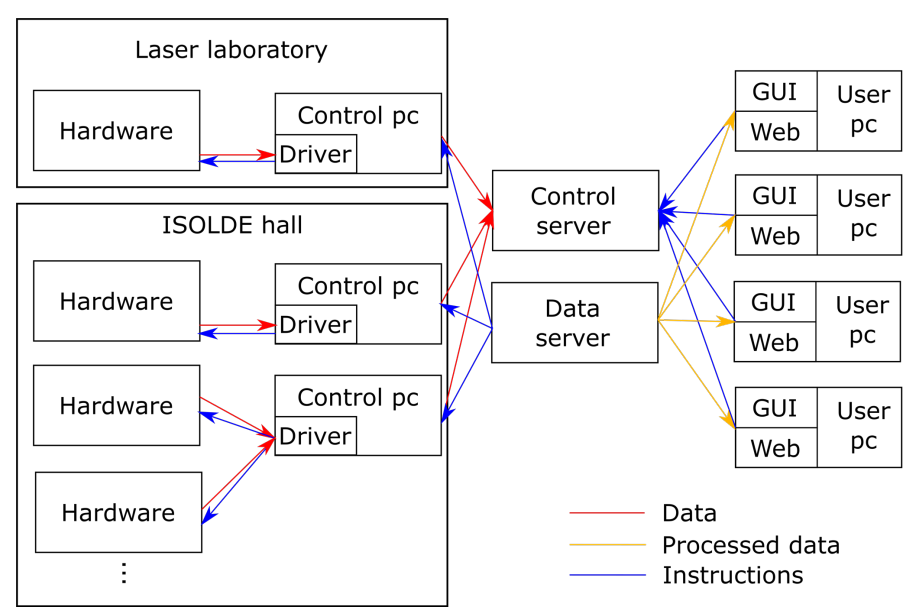

Figure 5: Diagram of the software architecture for the CRISTAL networked data-acquisition system

${ }^{1}$ Contact: ruben.p.degroote@jyu.fi 


\section{Background from long-lived high-lying states}

The introduction of a non-evaporable getter pump [26], ad- ${ }_{220}$ ditional differential pumping, and the new UHV CEC chamber has reduced the pressure to $3(1) \times 10^{-10}$ mbar in the interaction region. This value was measured with valves open to the CEC region at $9 \times 10^{-8}$ mbar, while the cell was loaded with $\mathrm{K}$ while the ends of the $\mathrm{CEC}$ were at $150^{\circ} \mathrm{C}$. This lower pressure helped reduce collisional background.

The charge-exchange process populates long-lived Rydberg ${ }_{225}$ and other high-lying states, creating a fraction of the atomic ensemble which have a higher cross-section for laser induced and collisional ionisation, significantly contributing to background [16]. Although it was initially thought that the deflector after

175 the CEC would ionize these states, field ionisation plates op- ${ }_{230}$ erated with an electric field strength of $5 \mathrm{kV} / \mathrm{cm}$ directly after the CEC were found to reduce this background rate by a factor of 3, for neutron deficient Sn neutralised (at masses 104120 ) by $K$ and a factor of 8 for neutron rich $K$ (at mass 41)

180 neutralised by K. A large proportion of this is due to isobaric ${ }_{235}$ and molecular contamination of the ion beam [27, 28] entering the CRIS beamline. In recent measurements at mass 130 , an isobaric contamination of $2 \times 10^{7}$ ions/s was observed from caesium (identified with the ISOLDE tape station [13]). While

185 during measurements of potassium at mass $52,1 \times 10^{7}$ ions/s of ${ }_{240}$ isobaric contamination was observed. A uranium carbide target was used in both cases. Using a second high power nonresonant $1064 \mathrm{~nm}$ laser pulse to ionize these states between the CEC and differential pumping, before the interaction region, has been found to reduce the photo-ionized background by $\mathrm{a}_{245}$ factor of 2 [12].

An evaluation of the background from these long-lived, highlying states can be seen in the simulation results of Figure 6. During the charge-exchange processes where ions are neutralised by electron capture from $\mathrm{K}$ or Na vapours, there is a crosssection for the exiting neutral atom to be left in an highlyexcited state. Even if the reaction has a large energy deficit the total fraction of these states populated can be significant due to the density of states near the continuum. The relative population of atomic states for which the ionisation potential of the element is within reach of Nd:YAG laser harmonics wavelengths are shown. These are typical non-resonant step laser wavelengths used at CRIS. These populations were simulated using a modified treatment by Rapp and Francis [24, 29] to calculate the cross-section for the initial populations, with final populations following $120 \mathrm{~cm}$ of flight at $40 \mathrm{keV}$. The deexcitation of the atoms was simulated using atomic transition data from NIST $[25,30]$. This was performed for ions of elements $1 \leq Z \leq 89$ neutralised by a $\mathrm{K}$ vapour. Although the atomic populations will be overestimated as many transition ${ }_{26}$ rates from these states are unknown, the relative population in the plot indicates the level of certainty one can have that these high-lying states will have a contribution to the background for a given element. For example for In $(Z=49)$ and its major iso-

215 baric contamination at ISOLDE for neutron-rich isotopes, $\mathrm{Cs}_{270}$ $(Z=55)$, this contribution can be expected to be significant for $1064 \mathrm{~nm}$ light.
A neutral beam [31] of alkali metal vapour from the CEC and molecular contamination may also contribute to the total background rate as it creates a volume along the atom beam path with an effectively worse vacuum, which would increase the collisional ionization rate. This contribution has not yet been experimentally evaluated.

\section{A simple universal ion-source arrangement}

A versatile ion optics arrangement has been constructed to allow compatibility with multiple types of ion source, and in principle to produce ion beams of all stable elements. A schematic diagram of the ion optics arrangement is shown in Figure 7 A). The arrangement was designed to have an electric field geometry which allows the extraction and reshaping of ions produced at both positions 1 . and 4.. Wide acceptance [32] extraction lenses at 8., 10. and steerers at 9., downstream improve the stability of the source over time and allow drifts to be compensated.

See Figure 7 B) for a diagram of the electrically isolated system used to control the high-voltages applied to the ion optics. The high-voltages are applied to the elements by 5 and $10 \mathrm{kV}$ Spellman MPS modular supplies controlled by a $0-10 \mathrm{~V}$ analog input. The analog control voltages are supplied from a 16-channel, 16-bit LTC2668 digital-to-analog converter floating at the ion source potential. This is controlled by SPI serial interface via a Linduino microcontroller, which has galvanic isolation to protect against shorting. The microcontroller is in turn controlled by serial commands issued from a Python interface by USB over a fiberoptic link to the high-voltage cage. The read-back of the high voltage supplies is also measured over this connection using a National Instruments USB-6008 analog-todigital converter. The ion sources, ion optics and high voltage supplies are held at a potential up to the supply voltage of $30 \mathrm{kV}$. Power is provided to the setup by a $240 \mathrm{~V}$ ac mains isolation transformer.

The use of a tantalum surface ion source $[33,34]$ in position 1. of Figure 7 A) allows effective ionization of elements with ionisation potentials below $6 \mathrm{eV}$ (the work function of Ta is $4.5 \mathrm{eV}$ ) [35], suitable for alkali and rare-earth metals. Laser ionisation scheme development with $\mathrm{K}$ was performed with this source before the online experiment, with energy spreads of typically $<0.1 \mathrm{eV}$ [36]. At a beam energy of $5 \mathrm{keV}$, a linewidth of $40 \mathrm{MHz}$ was reached.

Reversing the polarity of the extractor element 2. Ext. 1 in Figure 7 A) allows only neutral atoms to escape the surface source, enabling it to be used as an atomic beam unit [37] in combination with laser beam paths transverse to the atom beam direction.

A plasma ion source can also be used in position 1. (Figure 7) A), allowing ionisation of volatile species such as $\mathrm{H}, \mathrm{N}$ and $\mathrm{CO}_{2}$ with the use of a hot filament [38]. An electron impact plasma ion source was used which has energy spreads of $<0.1$ $\mathrm{eV}$ [39] and is capable of delivering $\mu \mathrm{A}$ beams. The use of a laser ablation ion source has shown the greatest utility for the experiment [23]. A target ladder of several materials is located in position 4. (Figure $7 \mathrm{~A}$ )) at $45^{\circ}$ to the laser windows and 


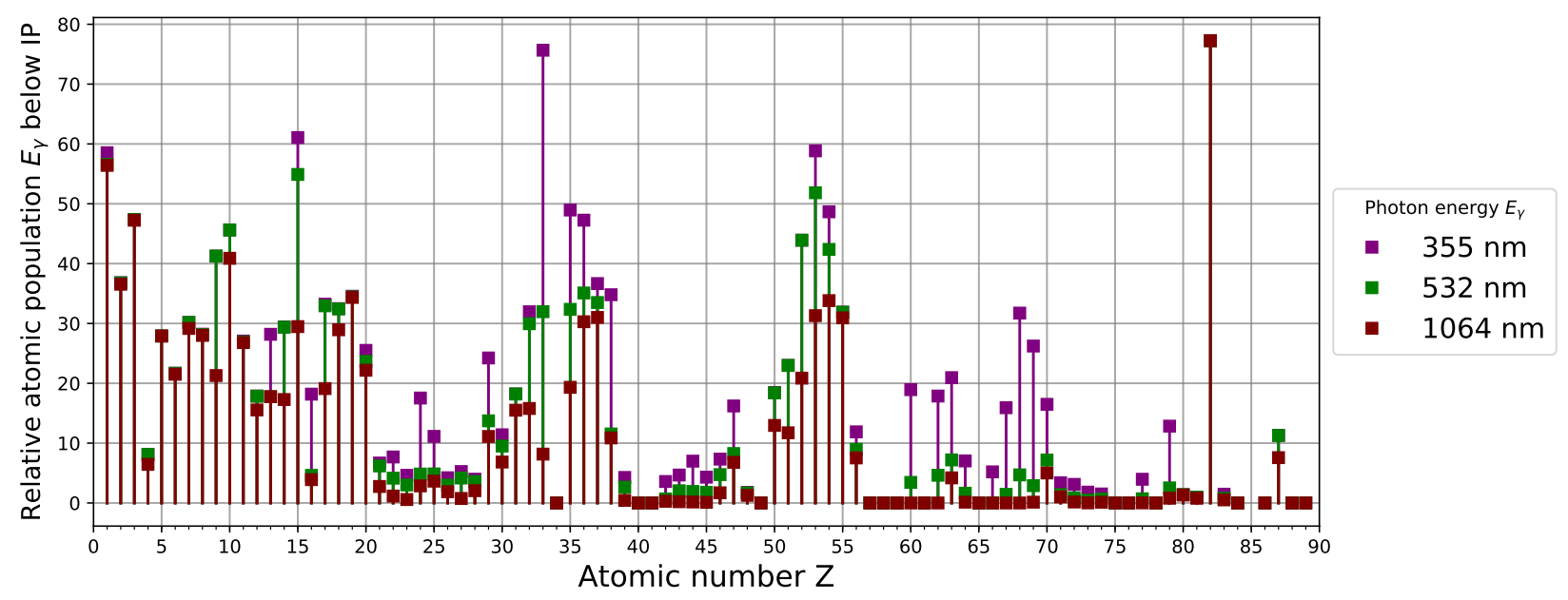

Figure 6: Calculated fraction of the atomic population within the reach of ionizing Nd:YAG laser photon energy, $\mathrm{E}_{\gamma}$, for elements Z $=1-89$. Cross-sections were calculated for a $40 \mathrm{keV}$ ion beam neutralised by $\mathrm{K}$ vapour using the treatment by [24]. Atomic transition data was taken from NIST [25], populations are after $120 \mathrm{~cm}$ of flight. Where insufficient line data was available only the initial population at $0 \mathrm{~cm}$ is given. No values are reported for elements with insufficient level information available.

A)

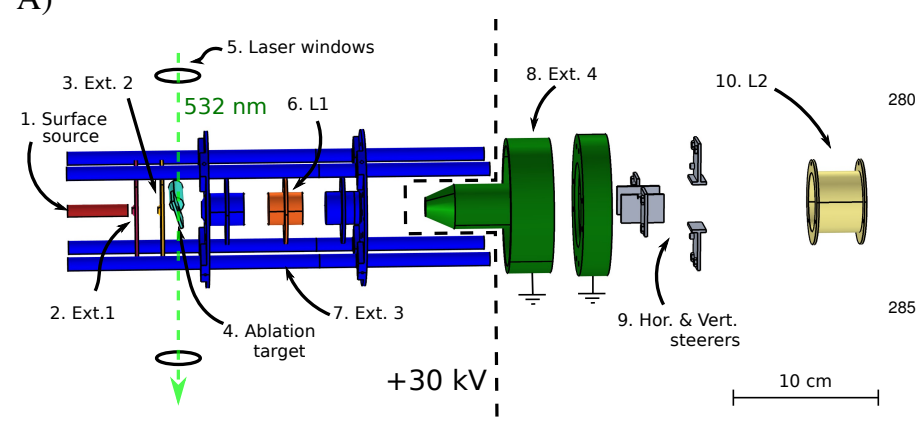

B)

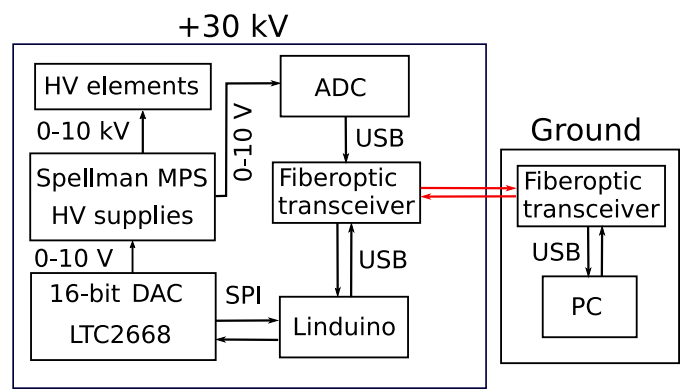

Figure 7: A) Elements at the high-voltage platform potential are indicated by ${ }_{300}$ the dashed line. B) Diagram of the isolated HV control setup. the ion beam path, allowing a pulsed 532-nm Nd:YAG (Litron LPY 601 50-100 PIV) laser to be focused onto a target with a spotsize of $<1 \mathrm{~mm}^{2}$. A typical fluence of $>0.5 \mathrm{~J} / \mathrm{cm}^{2}$ onto the target materials is used to create a laser-induced plasma for extraction by the ion optics. This corresponds to an average beam current of 50(2) pA into the interaction region of CRIS, after transmission out of the ion source arrangement through a $90^{\circ}$ electrostatic bender and then a $33^{\circ}$ electrostatic bender into the CRIS beamline.

The system reaches equilibrium and produces a stable beam current after 1 hour, depending upon the target surface and composition and the laser fluence used. It has been operated continuously for over a week. The ablation process is known to be complex and contains many regimes of ionisation [40, 41, 42]. Energy spreads of $0.1-1 \mathrm{keV}$ are typically observed in the ablation process [43]. However the ablation process gives the ideal pulsed time structure needed for CRIS, with a high ion bunch insity. An estimated $\sim 10^{6}$ ions/bunch at a rep-rate of $100 \mathrm{~Hz}$ have been measured for the ablation of In and Sn targets. At ablation laser pulse fluences of $<1 \mathrm{~J} / \mathrm{cm}^{2}$ very little information is available, due in part to the detection sensitivity limitations of other techniques. As demonstrated in our recent work 295 [23] on ${ }^{113,115} \mathrm{In}$, it is possible to achieve spectral linewidths of $150(20) \mathrm{MHz}$ with fluences $<1 \mathrm{~J} / \mathrm{cm}^{2}$ by using a configuration with a near zero electric field strength around the ablation plasma.

It was found to be possible to correct spectra to $40(10) \mathrm{MHz}$ resolution despite energy spreads of $0.1-3 \mathrm{eV}$, by using the timeof-flight data of the resonantly ionized ions recorded by the TimeTagger4-2G TDC. Gating on the different hyperfine transition frequencies of ${ }^{113,115}$ In also allowed completely resolvable time-of-flight peaks for each In isotope mass, as shown in 
selectivity of the atomic transitions. A selectivity of $2 \times 10^{4}$ was reached for ${ }^{113,115} \mathrm{In}$ at $20 \mathrm{keV}$ [23].

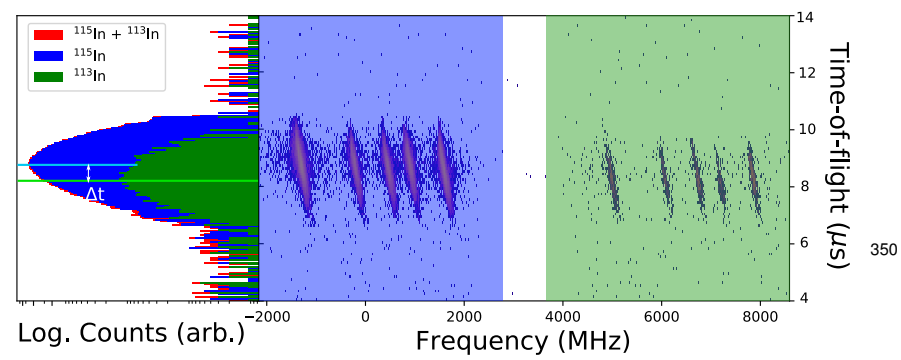

Figure 8: Gating on the frequency range of the hyperfine structures of ${ }^{113} \operatorname{In}_{355}$ or ${ }^{115}$ In completely resolves the time-of-flight of the two isotopes, allowing determination of their time-of-flight difference $\Delta t$. Figure modified from [23], see [23] for more details.

Ionisation by laser ablation allows the production of ion $^{360}$ beams of almost any element or molecule, provided it can be contained within a solid matrix to be ablated [44, 45]. Many initial charge states are produced and can be selected electrostatically [46]. This new source has supported the development ${ }^{365}$ of laser ionisation schemes of $\mathrm{In}, \mathrm{Sn}$ and $\mathrm{BaF}$.

\section{Outlook}

Further progress has been made in streamlining the CRIS setup since 2015 [2], and reaching its theoretical sensitivity and efficiency, through understanding the systematics of the technique and increasing the reliability of its already established operation.

Several areas remain to be fully characterised which may contribute to further improvement of the total experimental efficiency of CRIS. This includes i) efficient beam transport through the CEC, ii) increasing the effective number of alkali vapor atoms available for neutralisation, iii) long term stabilization of the spatial laser-atom overlap within the interaction region, ${ }_{38}$ iv) transport of the resonantly ionized atoms through $20^{\circ}$ to the detector and $v$ ) efficiency of the laser-ionisation schemes.

Other planned areas of investigation and improvements include i) continuous monitoring of atom and laser beam position ${ }_{390}$ and intensities, ii) the introduction of field ionisation of resonantly produced Rydberg states [16], iii) energy filtering of collisional ions, iv) ion ionisation and v) further optimization of the DSS setup.

\section{Acknowledgements}

This work was supported by ERC Consolidator Grant No.400 648381 (FNPMLS); STFC grants ST/L005794/1 and ST/L005786/1 and Ernest Rutherford Grant No. ST/L002868/1; GOA 15/010 from KU Leuven; BriX Research Program No. P7/12; the FWO-Vlaanderen (Belgium); Belgian Federal Science Policy ${ }^{405}$ Office (BELSPO); the European Unions Seventh Framework Programme for Research and Technological Development under Grant Agreement 654002 (ENSAR2); National Key R\&D
Program of China (Contract No:2018YFA0404403), the National Natural Science Foundation of China (No:11875073) and we acknowledge the financial aid of the Ed Schneiderman Fund at New York University.

We would also like to thank the ISOLDE collaboration and technical teams for their support.

\section{References}

[1] K. T. Flanagan, et al. Physical Review Letters, 111(21):212501, 2013. ISSN 0031-9007. URL http://link.aps.org/doi/10.1103/ PhysRevLett.111.212501.

[2] T. E. Cocolios, et al. Nuclear Instruments and Methods in Physics Research, Section B: Beam Interactions with Materials and Atoms, 376:284, 2016. ISSN 0168583X.

[3] W. H. Wing, et al. Physical Review Letters, 36(25):1488, 1976 ISSN 0031-9007. URL https://link.aps.org/doi/10.1103/ PhysRevLett.36.1488.

[4] S. Kaufman. Optics Communications, 17(3):309, 1976. ISSN 00304018. URL http://linkinghub.elsevier.com/retrieve/ pii/0030401876902674.

[5] P. Campbell, et al. Progress in Particle and Nuclear Physics, 86:127, 2016. ISSN 01466410. URL https://linkinghub.elsevier.com/ retrieve/pii/S0146641015000915.

[6] G. J. Farooq-Smith, et al. Physical Review C, 94(5):054305, 2016. ISSN 2469-9985. URL http://link. aps.org/doi/10.1103/PhysRevC. 94.054305 .

[7] S. G. Wilkins, et al. Physical Review C, 96(3):034317, 2017. ISSN 2469 9985. URL https://link.aps.org/doi/10.1103/PhysRevC.96. 034317.

[8] K. M. Lynch, et al. Physical Review C, 93(1):014319, 2016. ISSN 2469-9985. URL http://link.aps.org/doi/10.1103/PhysRevC. 93.014319.

[9] K. M. Lynch, et al. Physical Review C, 97(2):1, 2018. ISSN 24699993.

[10] G. J. Farooq-Smith, et al. Physical Review C, 96(4):044324, 2017. ISSN 2469-9985. URL https://link.aps.org/doi/10.1103/ PhysRevC.96.044324.

[11] R. P. De Groote, et al. Physical Review C, 96(4):1, 2017. ISSN 24699993.

[12] A. Koszorus. Nuclear Instruments and Methods in Physics Research Section B: Beam Interactions with Materials and Atoms, Conference(EMIS2018), 2019.

[13] R. Catherall, et al. Journal of Physics G: Nuclear and Particle Physics, 44(9):094002, 2017. ISSN 0954-3899. URL http: //stacks. iop.org/0954-3899/44/i=9/a=094002?key= crossref . cb9ee490ac00453f72e007300938b2c6.

[14] E. Mané, et al. European Physical Journal A, 42(3):503, 2009. ISSN 14346001.

[15] S. Paulo. Nuclear Instruments and Methods, 179:195, 1981.

[16] C. Schulz, et al. Journal of Physics B: Atomic, Molecular and Optical Physics, 24(22):4831, 1991. ISSN 0953-4075. URL http://stacks.iop.org/0953-4075/24/i=22/a=020?key= crossref. 2c87f8c494d942077faa53dbed 7374 f5.

[17] V. Sonnenschein, et al. Laser Physics, 27(8):085701, 2017. ISSN 1054-660X. URL http: //stacks. iop.org/1555-6611/27/i=8/a= 085701?key=crossref . ae700ab1aa5d94924db40c8ed77f ebe 7 .

[18] R. P. de Groote, et al. Physical review letters, 115(13):132501, 2015. ISSN 1079-7114. URL http://journals .aps .org/prl/abstract/ 10.1103/PhysRevLett.115.132501.

[19] K. Lynch, et al. Nuclear Instruments and Methods in Physics Research Section A: Accelerators, Spectrometers, Detectors and Associated Equipment, 844:14, 2017. ISSN 01689002.

[20] K. M. Lynch, et al. Hyperfine Interactions, 216(1-3):95, 2013. ISSN 03043843.

[21] K. M. Lynch, et al. Physical Review X, 4(1):1, 2014. ISSN 21603308. URL http://link. aps .org/doi/10.1103/PhysRevX .4.011055.

[22] X. Huang, et al. Nuclear Instruments and Methods in Physics Research, Section A: Accelerators, Spectrometers, Detectors and Associated Equipment, 726:77, 2013. ISSN 01689002. 
[23] R. F. Garcia Ruiz, et al. Physical Review X, 8(4):041005, 2018.480 ISSN 2160-3308. URL https://link.aps.org/doi/10.1103/ PhysRevX .8.041005.

[24] D. Rapp et al. The Journal of Chemical Physics, 37(11):2631, 1962. ISSN 00219606. URL http://scitation.aip.org/content/aip/ journal/jcp/37/11/10.1063/1.1733066.

[25] N. A. T. A. Kramida, Y. Ralchenko, J. Reader. NIST Atomic Spectra Database, 2014. URL http://www .nist.gov/pml/data/asd.cfm.

[26] C. Benvenuti, et al. Vacuum, 53(1-2):219, 1999. ISSN 0042-207X. URL https://www.sciencedirect.com/science/article/pii/ S0042207X98003777.

[27] A. Gottberg, et al. Nuclear Instruments and Methods in Physics Research Section B: Beam Interactions with Materials and Atoms, 336:143, 2014. ISSN 0168-583X. URL https://www.sciencedirect.com/ science/article/pii/S0168583X1400528X.

[28] D. Fink, et al. Nuclear Instruments and Methods in Physics Research Section B: Beam Interactions with Materials and Atoms, 344:83, 2015. ISSN 0168-583X. URL https://www.sciencedirect.com/ science/article/pii/S0168583X14010222.

[29] A. Vernon, et al. Spectrochimica Acta Part B: Atomic Spectroscopy, 153:61, 2019. ISSN 0584-8547. URL https : //www. sciencedirect. com/science/article/pii/S0584854718305597.

[30] C. Ryder, et al. Spectrochimica Acta Part B: Atomic Spectroscopy, 113:16, 2015. ISSN 05848547. URL http://linkinghub.elsevier . com/retrieve/pii/S0584854715001962.

[31] S. Zhang, et al. Physics Procedia, 32:513, 2012. ISSN 18753892. URL https://www. sciencedirect.com/science/article/ pii/S1875389212010127.

[32] J. E. Delmore. A WIDE APERTURE ION LENS Tech. rep., 1984. URL https://ac.els-cdn.com/ 0168117684850399/1-s2.0-0168117684850399-main.pdf? $\left\{\_\right\}$tid=c4004689-6b45-4bc9-9304-5aeeb2d1274a $\{\&\}$ acdnat $=$ 1538044038\{_\}9607cead66bb4e4f58e2d2c0981ed62b.

[33] D. M. Jamba. Review of Scientific Instruments, 40(8):1072, 1969. ISSN 0034-6748. URL http://aip.scitation.org/doi/10.1063/ 1.1684155.

445 [34] J. B. Hasted. Physics of atomic collisions. American Elsevier, 2d ed. ed. 1972. URL https://bibdata.princeton.edu/bibliographic/ 1917772.

[35] J. Lettry, et al. Nuclear Instruments and Methods in Physics Research Section B: Beam Interactions with Materials and Atoms, 204:363, 2003. ISSN 0168-583X. URL https://www.sciencedirect.com/ science/article/pii/S0168583X02019675.

[36] R. Kirchner. Nuclear Instruments and Methods in Physics Research Section A: Accelerators, Spectrometers, Detectors and Associated Equipment, 292(2):203, 1990. ISSN 0168-9002. URL https://www. sciencedirect.com/science/article/pii/016890029090377I.

[37] F. Gao, et al. AIP Advances, 4(2):027118, 2014. ISSN 2158-3226. URL http://aip.scitation.org/doi/10.1063/1.4866983.

[38] H. Conrads et al. Plasma Sources Science and Technology, 9(4):441, 2000. ISSN 0963-0252. URL http: //stacks. iop.org/0963-0252/9/i=4/a=301 ?key=crossref . $4 \mathrm{fd} 3 \mathrm{f} 4207$ ddcb8c7bfc482edfbe7b293.

[39] J. Blais et al. International Journal of Mass Spectrometry and Ion Physics, 20(2-3):329, 1976. ISSN 0020-7381. URL https://www . sciencedirect.com/science/article/pii/0020738176801593.

[40] L. Torrisi, et al. Applied Surface Science, 210:262, 2003. URL http://web3.le.infn.it/sub-web/leas/Pubblicazioni/ COMPARISONOFNANOSECONDLASERABLATION .pdf.

[41] J. Maul, et al. Physical Review B, 71(4):045428, 2005. ISSN 10980121. URL https://link.aps.org/doi/10.1103/PhysRevB.71. 045428.

[42] K. K. Anoop, et al. Article in Journal of Applied Physics, 2015. URL https://www.researchgate.net/publication/272815013.

[43] X. Wang, et al. Spectrochimica Acta Part B: Atomic Spectroscopy, 99:101, 2014. ISSN 0584-8547. URL https : //www.sciencedirect. com/science/article/pii/S0584854714001220.

[44] S. Olmschenk et al. Applied Physics B, 123(4):99, 2017. ISSN 0946-2171. URL http://link.springer.com/10.1007/ s00340-017-6683-1.

[45] B. N. Chichkov, et al. Applied Physics A Materials Science E Processing,
63(2):109, 1996. ISSN 0947-8396. URL http://link.springer. com/10.1007/BF01567637.

[46] M. Kumaki, et al. Review of Scientific Instruments, 87(2):02A921, 2016. ISSN 0034-6748. URL http: //aip.scitation.org/doi/10.1063/ 1.4939781. 